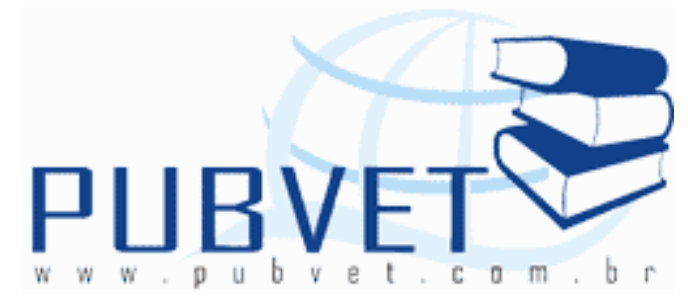

PUBVET, Publicações em Medicina Veterinária e Zootecnia.

\title{
Acupuntura como opção para analgesia em veterinária
}

Natali Silva Braga ${ }^{1}$, Angélica do Rocio Carvalho Silva ${ }^{2}$

${ }^{1}$ Médica Veterinária autônoma - nattysilvabraga@yahoo.com.br

2 Doutora - Docente de Ensino Superior - Cirurgia de Pequenos Animais e Metodologia Cientifica - UNIFEOB (São João Da Boa Vista) e FESB (Bragança Paulista)

\section{Resumo}

Dor é uma resposta protetora do organismo a estímulos nocivos, que resulta em efeitos indesejáveis quando não controlada. O reconhecimento da dor é importante para a instituição de uma terapia analgésica eficiente, pois a síndrome dolorosa pode ser considerada uma doença, gerando alterações na homeostasia orgânica que implicam em perda da qualidade de vida do paciente. A analgesia pode ser promovida mediante a utilização de vários tipos de fármacos. No entanto, esses podem causar efeitos adversos de acordo com a espécie e condição física do paciente. A acupuntura, que atua sobre o controle da dor por ativação de vias opióides e não opióides, tem se mostrado eficaz como uma opção que, gerando a hipoalgesia, pode melhorar a condição de bem-estar do animal, além de poder ser utilizada como recurso coanalgésico pela capacidade de diminuir a quantidade de fármacos utilizados para o controle da dor, sendo raramente contraindicada.

Palavras-chave: analgesia, hipoalgesia, bem-estar animal, Medicina Tradicional Chinesa, cães. 
BRAGA, N.S. e SILVA, A.R.C. Acupuntura como opção para analgesia em veterinária. PUBVET, Londrina, V. 6, N. 28, Ed. 215, Art. 1435, 2012.

\title{
Acupuncture as an option for veterinary analgesia
}

\begin{abstract}
Pain is a protective response from the body to harmful stimulation that results in undesirable effects when not controlled. Pain recognition is important to the institution of an efficient painkiller therapy because the pain syndrome can be considered a disease, generating alterations in the organic homeostasis that imply in lost of quality life of the patient. The analgesia can be promoted by the use of several kinds of drugs. However, these can cause side effects according to species and physical condition of the patient. Acupuncture acts to control pain by activation of the opioids pathways and not opioids and this method has been proven effective as an option that, generating hypoalgesia, can improve the animal's welfare besides being used and an painkiller adjuvant resource for the ability to decrease the amount of druds used to control the pain and is rarely contraindicated.
\end{abstract}

Keywords: painkiller, hypoalgesia, animal welfare, traditional Chinese medicine, dog.

\section{INTRODUÇÃO}

Nos últimos anos, tanto os Médicos Veterinários como a sociedade em geral, tem voltado mais sua atenção para os problemas de dor em animais. A Associação Internacional para o Estudo da Dor (AIED) definiu a dor como "experiência sensorial e/ou emocional desagradável associada a um dano tecidual real, ou potencial". Embora esta definição seja estabelecida para pacientes humanos, hoje em dia é uniformemente aplicada também a animais submetidos a estímulo nociceptivo ou doloroso (HELLEBREKERS, 2002a).

No dia-a-dia, os veterinários e as pessoas que tomam conta de animais usam observações comportamentais para avaliar o bem-estar daqueles que se encontram sob sua proteção. A dor é uma experiência individual que depende de alguns fatores para ser observável e mensurável. Algumas dessas variáveis 
BRAGA, N.S. e SILVA, A.R.C. Acupuntura como opção para analgesia em veterinária. PUBVET, Londrina, V. 6, N. 28, Ed. 215, Art. 1435, 2012.

são: a espécie, a linhagem genética dentro da espécie, o sexo, o peso corpóreo, o condicionamento prévio, a dominância social do animal, a saúde em geral e as condições do meio ambiente no momento da observação (HARDIE, 2002).

Portanto, a dor pode ser classificada de acordo com as sensações (as quais só podem ser registradas através de uma observação detalhada e da palpação no animal) e do estado do animal (após o questionamento do dono) (DRAEHMPAEHL; ZOHMANN, 1997). Independentemente da forma de classificação, é muito importante a identificação e o controle da dor em animais, já que a dor diminui o bem-estar dos mesmos.

$\mathrm{Na}$ terapia analgésica geralmente são usados fármacos, porém estes podem apresentar efeitos indesejáveis e riscos aos animais (FANTONI; MASTROCINQUE, 2002).

Entre as terapias complementares para o controle da dor, a acupuntura vem se mostrando eficaz, já que a terapia da dor representa o campo propriamente dito da acupuntura, pois a acupuntura leva um estado de equilíbrio no Sistema Nervoso do animal. (DRAEHMPAHEL; ZOHMANN, 1997).

O objetivo desse estudo bibliográfico é abordar a fisiopatologia da dor e o controle da mesma por meio da acupuntura.

\section{FISIOPATOLOGIA DA DOR}

A dor tem sido considerada como importante mecanismo de proteção para o corpo porque provoca, no indivíduo, uma reação para remover o estímulo doloroso (FANTONI; MASTROCINQUE, 2002).

O primeiro tipo de dor é a dor fisiológica (Figura 1), que desempenha um papel adaptativo, como um dos mecanismos de defesa normal do corpo, que avisa quando há algo prejudicial, ou insultos ambientais, e inicia estratégias de prevenção comportamentais e de reflexas (LAMONT et al., 2000). 


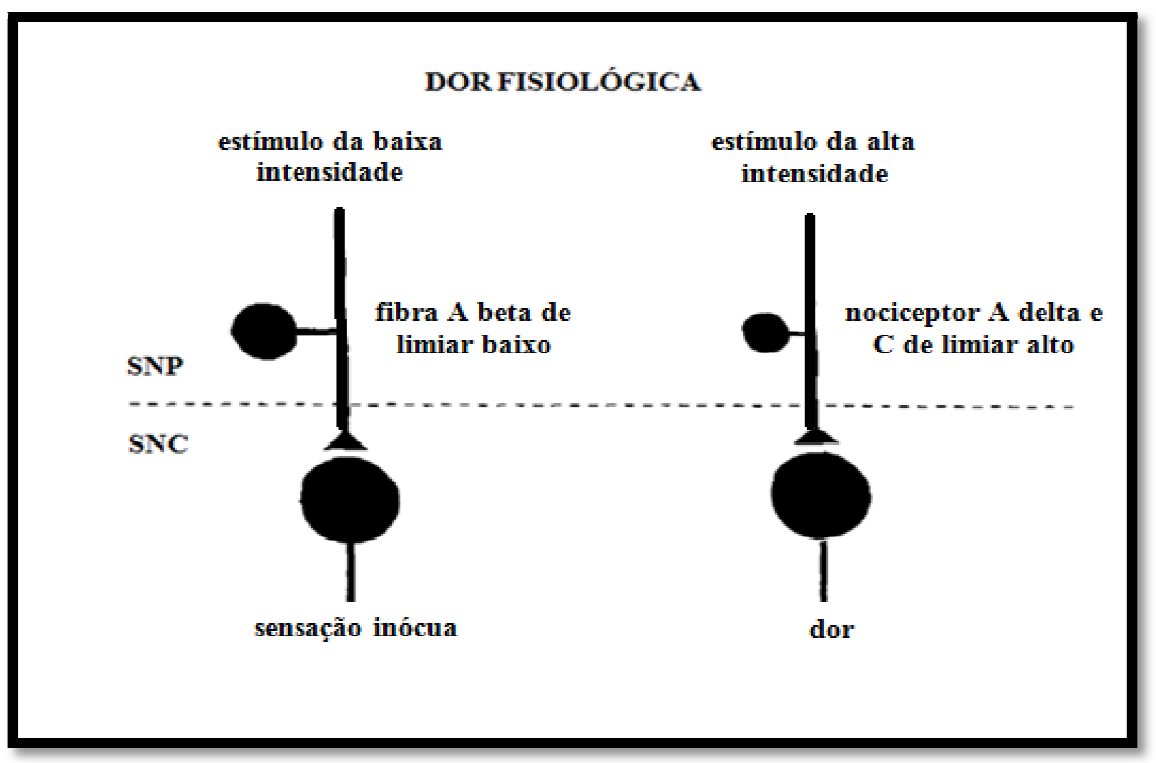

Figura 1. Dor fisiológica. Em condições fisiológicas, o estimulo de baixa intensidade resulta em um estimulo não nocivo (tátil), ao passo que o estímulo de alta intensidade, depois da ativação do nociceptor e da propagação do sinal por vias aferentes separadas, pode provocar a dor.

SNP - sistema nervoso periférico; SNC - sistema nervoso central Fonte: HELLEBREKERS, 2002b

O termo nocicepção está relacionado com o reconhecimento pelo sistema nervoso, de sinais que se originam em receptores sensoriais (nociceptores) e que fornecem informações relacionadas ao dano tissular (HELLEBREKERS, 2002b; LAMONT et al., 2000).

Quando esses receptores são estimulados, as terminações nervosas sensoriais livres são ativadas e, dependendo do tipo de estimulação, o potencial de ação é transportado centralmente por uma classe especifica de fibras. Os nociceptores que respondem à estimulação térmica ou mecânica possuem diâmetro pequeno e são fibras mielinizadas (do tipo A delta) que transportam sinais de alta velocidade (5 a $30 \mathrm{~m} / \mathrm{s}$ ) (Figura1) (HELLEBREKERS, 2002b).

Os outros nociceptores compõem o grupo dos polimodais, que podem ser estimulados de diversas formas, como estímulos intensos de natureza química, 
BRAGA, N.S. e SILVA, A.R.C. Acupuntura como opção para analgesia em veterinária. PUBVET, Londrina, V. 6, N. 28, Ed. 215, Art. 1435, 2012.

térmica (calor ou frio) ou mecânica. Os sinais provenientes desses receptores são transportados por fibras aferentes de uma classe diferente (fibras do tipo C) (Figura 1), de diâmetro pequeno, não-mielinizadas, com uma velocidade de condução de 0,5 a $2 \mathrm{~m} / \mathrm{s}$. Durante a ativação, essas fibras intensificam a atividade do estímulo e serão responsáveis pela dor fraca e prolongada. (HELLEBREKERS, 2002b).

Os nociceptores dos músculos são, primeiramente, mediados pelas fibras C polimodais e estimulados por substâncias como a bradicinina, histamina, serotonina ou cloreto de potássio e respondem, também, à forte pressão. Os nociceptores A-delta e C constituem plexos de cápsulas articulares, ligamentos e membranas adventícias dos vasos sanguíneos que irrigam as articulações. Estes nociceptores são sensíveis a estímulos mecânico e químico, incluindo, também, a sensibilização por prostaglandinas (COGGESHALL et al., 1983).

Em sua forma mais simples, o caminho do estímulo nociceptivo pode ser considerado como uma cadeia de três neurônios: neurônios de primeira ordem originários da periferia e projetando para a coluna vertebral, neurônios de segunda ordem ascendente da medula espinhal e os de terceira ordem que são os que se projetam para o córtex cerebral (Figura 2) (LAMONTet al., 2000). 


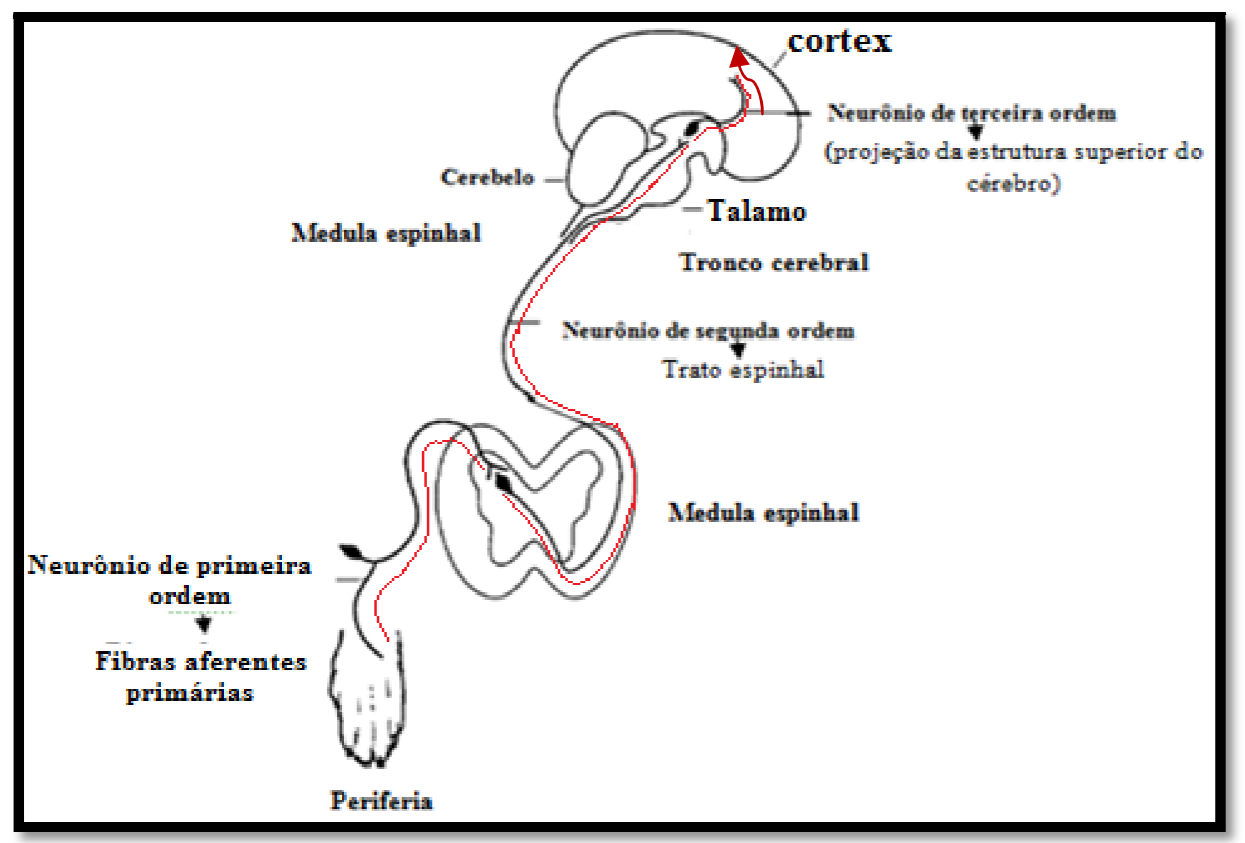

Figura 2. Representação simplificada do processamento nociceptivo como uma cadeia de três neurônios. Um estímulo nocivo na periferia, ativa uma fibra aferente primária que transmite a informação para o corno dorsal da medula espinhal. $\mathrm{Na}$ medula espinhal um neurônio de projeção de segunda ordem que sobe no trato espinhal ao nível do tálamo intervém. Um terceiro neurônio transmite o estímulo nocivo modificado para os centros cerebrais superiores (cerebral córtex) para a percepção.

Fonte: Modificado de LAMONT et al., 2000.

As fibras aferentes primárias, que se localizam partir da periferia, adentram o canal espinhal, por meio da raiz dorsal, como nervos periféricos, para o interior do corno dorsal da medula espinhal, onde os sinais são posteriormente processados. As fibras A-delta, por exemplo, conectam-se, por meio de interneurônios, com os neurônios motores que são responsáveis pela resposta da retração reflexa. Junto com esses reflexos segmentais, as informações sensoriais relevantes para a sensação de dor são transportadas, por meio de vias específicas, para as áreas distintas do cérebro, como na região talâmica e na formação reticular. A partir disso, as informações são retransmitidas para o córtex sensorial, onde ocorre a experiência consciente (HELLEBREKERS,2002b). 
BRAGA, N.S. e SILVA, A.R.C. Acupuntura como opção para analgesia em veterinária. PUBVET, Londrina, V. 6, N. 28, Ed. 215, Art. 1435, 2012.

A transmissão discriminativa da dor faz o caminho da medula espinhal para as estruturas encefálicas e é realizada por meio dos tratos neoespinotalâmico, pela coluna dorsal pós-sináptica e pelo espinocervicotalâmico. Na coluna dorsal localizam-se grossas fibras em conjunto que constituem o "Controle Central do Gatilho", que tem a função de ativar rapidamente o processo do controle central para o córtex, anteriormente à chegada das informações nociceptivas ascendentes (BENSON et al., 1996 apud ESPER, 2005).

O "Controle Central do Gatilho" é uma teoria que foi proposta por Melzaj e Wall, em 1965, que afirma que os milhões de receptores do corpo conservam o cérebro abastecido de informações sobre temperatura e condições dos tecidos e órgãos. Os receptores e o sistema nervoso central comunicam-se por meio de um complexo código neural, que compreende a atividade relativa de fibras grossas e fibras finas. As fibras mais grossas ou $A \beta$ transmitem impulsos como os originados nos receptores do tato; as mais finas, do tipo C e A delta, de transmissão maislenta, conduzem os impulsos de dor (THURMON et al., 1996 apud KLAUMANN, et al., 2008).

Quando se arranha a pele suavemente, as fibras grossas conduzem impulsos que são percebidos, porém não traduzem uma sensação desagradável, pois a "porta" conserva-se fechada.Se a pele continuar a ser arranhada, cada vez com maior intensidade, mais receptores são estimulados e as fibras grossas sobrecarregadas fazem com que a "porta" se abra e as fibras finas transmitem impulsos dolorosos aos centros nervosos superiores (sensibilização central) (THURMON et al., 1996 apud KLAUMAN et al., 2008).

A dor fisiológica, por si só é rara na prática da clínica. Na maioria das situações, o estimulo nocivo não é transitório e pode estar associado à inflamação do tecido e lesão do nervo. Este tipo de dor é chamado de dor patológica (implica que o dano tecidual já ocorreu), ou dor clínica, como por exemplo, o desconforto e asensibilidade anormal que são características da sintomatologia clínica do paciente (Figura 3) (LAMONT et al., 2000). 


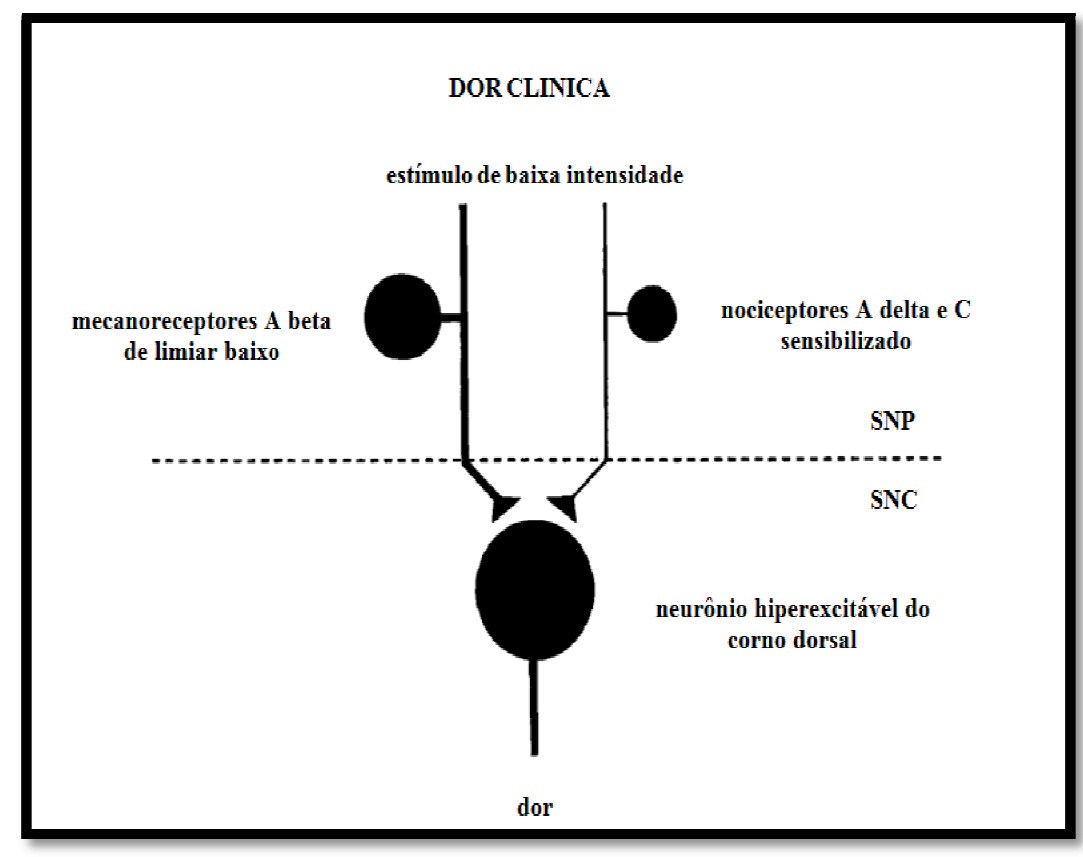

Figura 3. Dor clínica: em um estado de dor, a alteração do processamento das informações sensoriais no SNP e no SNC, e subsequente hiperexcitabilidade, resulta em estimulos de baixa intensidade que são, no momento, percebidos como dolorsos.

SNP - sistema nervoso periférico; SNC - sistema nervoso central.

Fonte: Modificado de LAMONT et al., 2000.

A dor patológica pode se manifestar de várias formas: dor espontânea que pode ser maçante, ardor (causalgia); dor exagerada em resposta a um estímulo nocivo(hiperalgesia); dor e produzida por um estímulo que normalmente não é nocivo(alodinia). Ela pode surgir de lesões a uma variedade de tecidos diferentes invocando mecanismos neurais, e muitas vezes é classificada em dor inflamatória (envolvendo estruturas somáticas ou viscerais) ou dor neurologica (envolvendo lesões do sistema nervoso). Além disso, é útil para caracterizar dor clínica a partir de uma perspectiva de duração e fazer a distinção entre dor aguda e de longa duração (crônica) (LAMONT et al., 2000). 
BRAGA, N.S. e SILVA, A.R.C. Acupuntura como opção para analgesia em veterinária. PUBVET, Londrina, V. 6, N. 28, Ed. 215, Art. 1435, 2012.

\section{Classificação da dor}

A classificação da dor é útil na área médica, pois fornece ao clínico,informações sobre sua possível origem e dirige os cuidados de saúde no sentido de um apropriado planejamento terapêutico farmacológico (DUARTE, 1998 apud ALMEIDA et al.,2006).

É importante destacar, no entanto, que a dor pode se modificar de acordo com a postura, atividade física, estado de atenção, emoções, temperatura, umidade ambiental, entre outros (CAPONERO et al., 2001).

A dor pode ser classificada quanto ao período de duração que pode ser dor aguda ou crônica; quanto à origem, sendo dor fisiológica e dor patológica; quanto à neurofisiologia, podendo ser dor nociceptiva e não nociceptiva (LAMONTet al., 2000).

A dor clínica aguda geralmente surge de um trauma de tecido mole ou inflamação, sendo o exemplo mais comum é dor cirúrgica pós-operatória. Apesar de não servir como uma função protetora, como a dor fisiológica, a dor aguda tem um papel biologicamente adaptativo, facilitando a reparação tecidual e cicatrização (LAMONT et al., 2000). Este tipo de dor apresenta-se bem delineada temporalmente e está associada a alterações neurovegetativas, tais como: taquicardia, hipertensão arterial, sudorese, palidez, expressão facial de intenso desconforto, agitação psicomotora e ansiedade (TEIXEIRA; PIMENTA, 1994 apud ESPER, 2005).

A dor crônica pode ser considerada como a dor que persiste além do tempo razoável para a cura de uma lesão, ou a que está associada a processos patológicos crônicos, que causam dor contínua ou recorrente, em intervalos de meses ou anos (LAMONT, et al., 2000).

A dor fisiológica, como descrita anteriormente, é o resultado do reconhecimento de um estímulo nocivo, que pode ser por calor, frio ou pressão. A dor patológica, que também foi já descrita, caracteriza-se pela transformação de um estímulo não lesivo em um estímulo lesivo, 
BRAGA, N.S. e SILVA, A.R.C. Acupuntura como opção para analgesia em veterinária. PUBVET, Londrina, V. 6, N. 28, Ed. 215, Art. 1435, 2012.

desencadeando sensibilidade dolorosa (alodínia), resposta exagerada aos estímulos nocivos (hiperalgesia) e aumento da área de hipersensibilidade. A dor patológica pode ser dividida em nociceptiva e não nociceptiva (FANTONI; MASTROCINQUE, 2002).

A dor nociceptiva, que pode ser visceral ou somática, resulta da ativação das terminações nervosas sensitivas delta $A$ e $C$ (nociceptores) por estímulos mecânicos, térmicos ou químicos. Esses processos podem ser sensibilizados por substâncias algogênicas, gerando como resultado a hiperalgesia (TRANQUILLI, 2004 apud ALMEIDA et al., 2006).

A dor não nociceptiva é dividida em neuropática e psicogênica. A dor neuropática é aquela cuja origem é a lesão, ou a irritação do nervo. Persiste por longo tempo após o evento precipitante e pode ser ocasionada por sensibilização central. Estímulos inócuos podem ser percebidos como dolorosos (alodínia). A dor psicogênica ocorre quando nenhum mecanismo nociceptivo ou neuropático pode ser identificado e há sintomas psicológicos suficientes para estabelecer critérios de distúrbios dolorosos. (DUARTE, 1998 apud ALMEIDA et al., 2006).

\section{DOR E BEM-ESTAR ANIMAL (BEA)}

O bem-estar de um indivíduo é seu estado em relação às suas tentativas de adaptar-se ao seu ambiente (BROOM, 1986).

Segundo o Animal Welfare Council (2009), o BEA é avaliado através das cinco liberdades que são: livre de fome e sede; livre de desconforto; livre de dor, ferimentos e doenças; livre de medo e angustia; livre para expressar seu comportamento natural.

De acordo com a bióloga Dra. Victoria Braithwaite, em entrevista à comissão do Prêmio WSPA (World Society for the Protection of Animals) de Bemestar Animal 2010, atualmente se acredita que muitas espécies, além dos vertebrados, também são sencientes. Compreendendo-se que senciência é a capacidade de vivenciar sentimentos e emoções, ou seja, ter a capacidade de 
BRAGA, N.S. e SILVA, A.R.C. Acupuntura como opção para analgesia em veterinária. PUBVET, Londrina, V. 6, N. 28, Ed. 215, Art. 1435, 2012.

sentir dor e de sofrer, assim como o oposto também é verdadeiro (um animal senciente também tem a capacidade de sentir prazer).

Portanto,o BEA é aplicado em prol da qualidade de vida dos animais e diz respeito ao bem estar físico e mental do animal (RAMOS, 2006).

Uma das formas de prejuízo do bem-estar de um animal é quando este sente dor, o que pode ser observado através de aspectos negativos específicos. Quando um animal sente dor, a cura de uma lesão é prejudicada por meio da ativação da resposta ao estresse. Quando o estresse é prolongado, a imunidade do animal diminui, aumentando a incidência de complicações. E se a dor é manifestada por um período longo, ela pode assumir um caráter crônico ficando mais difícil de ser tratada (HELLEBREKERS, 2002a).

Doença, ferimento, dificuldades de movimento e anormalidades de crescimento são todos indicativos de baixo grau de bem-estar. O bem-estar de um animal doente é sempre mais pobre que o bem-estar de um animal que não está doente. A dor pode será avaliada pela análise da aversão ao toque, dos parâmetros fisiológicos, dos efeitos de analgésicos, pela existência de neuromas, entre outras observações (GENTLE, 1986 apud BROOM, 1986).

Existem alguns métodos para identificar o grau de dor em animais que são as escalas de dor. A escala descritiva simples possui de três a cinco graduações e descrições do que cada algarismo representa (Quadro 1). Nesse caso o pesquisador observa o animal e escolhe um número que equivale o grau de dor que o animal esta sentindo (HARDIE, 2002).

As escalas analógicas visuais são linhas de comprimento padronizado, com nenhuma dor, de um lado; e a pior dor possível do outro (Quadro 2). Com frequência são elaboradas descrições comportamentais do que se observa entre os dois extremos da linha. O observador desenha uma linha no ponto em que ele julga haver dor. A distância da ausência de dor até a marca é dividida pelo comprimento total da linha, fornecendo a escala de dor (HARDIE, 2002). 
BRAGA, N.S. e SILVA, A.R.C. Acupuntura como opção para analgesia em veterinária. PUBVET, Londrina, V. 6, N. 28, Ed. 215, Art. 1435, 2012.

Quadro 1. Exemplos de escalas descritivas simples, utilizadas para graduar a dor em animais. $O$ item A é mais utilizado para equinos, e a escala vai de 0 a 4 , onde 0 quer dizer que o animal marcha normal e 4 o animal apresenta uma claudicação mesmo sem carregar peso. $O$ item $B$ pode ser utilizado para qualquer espécie e a escala vai de 0 a 4 , onde 0 significa que o animal não apresenta dor e 4 o animal apresenta pior dor possível. E o item $C$ também pode ser utilizado para animais de qualquer espécie e a escala vai de 0 a 3, onde 0 significa que o animal não apresenta nenhuma dor e 3 o animal apresenta uma dor grave.

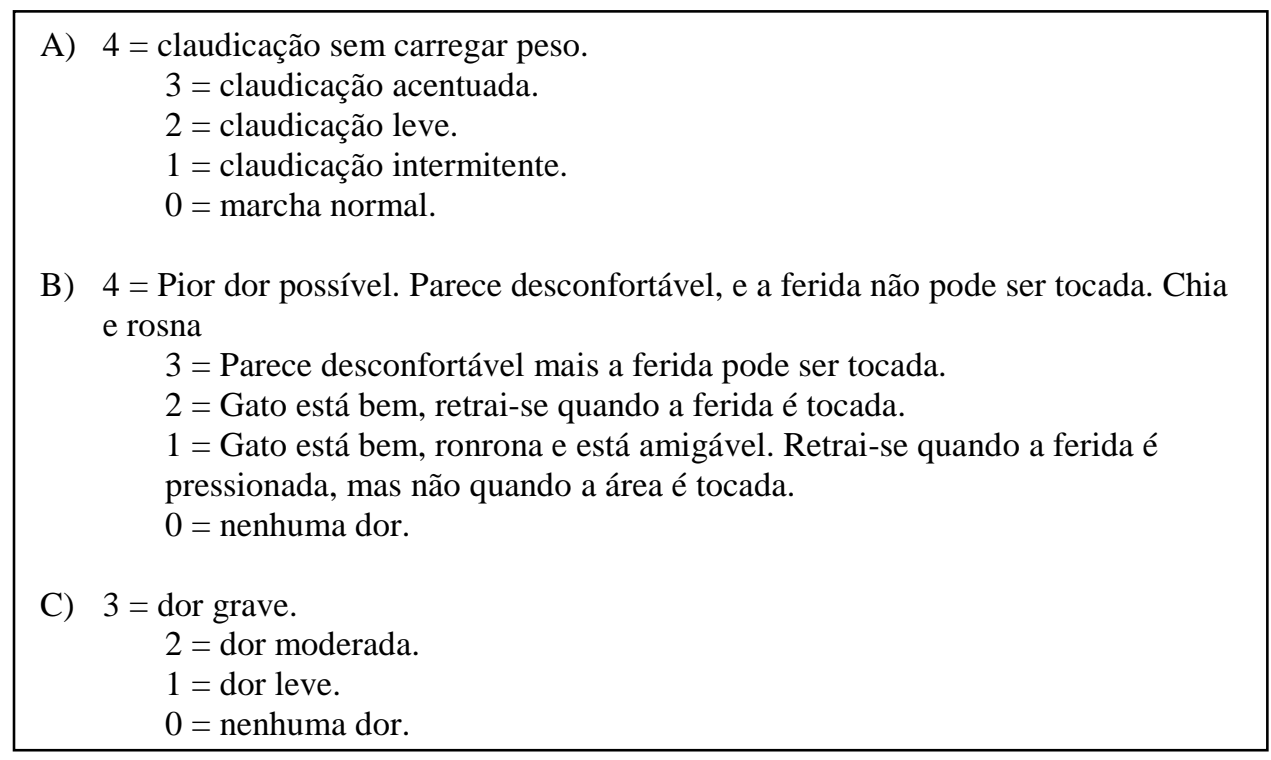

Fonte: HARDIE, 2002.

Quadro 2. Exemplos de escalas analógicas visuais utilizadas para graduar a dor em animais. Em uma ponta esta NENHUMA DOR e na outra ponta esta A PIOR DOR POSSÍVEL. O observador vai desenhar uma linha no ponto onde ele acha que o animal sente dor entre NENHUMA DOR e A PIOR DOR POSSÍVEL. Da mesma maneira é para ser feito com SADIO e com A PIOR INCAPACIDADE POSSIVEL.

Nenhuma dor A pior dor possível

Sadio A pior incapacidade possível

Fonte: HARDIE, 2002. 
BRAGA, N.S. e SILVA, A.R.C. Acupuntura como opção para analgesia em veterinária. PUBVET, Londrina, V. 6, N. 28, Ed. 215, Art. 1435, 2012.

A escala de contagem numérica é semelhante à escala analógica da dor, mas a diferença é que na escala numérica o observador escolhe de 0-10 ou 0100 (Quadro 3) (HARDIE, 2002).

Quadro 3. Exemplo de uma escala numérica para graduar a dor em animais. O Observador vai escolher um número para medir a dor no animal, 0 quer dizer que o animal não apresenta dor e 10 o animal apresenta a pior dor possível.

$\begin{array}{llllllllllll}\text { Nenhuma dor } & 0 & 1 & 2 & 3 & 4 & 5 & 6 & 7 & 8 & 9 & 10 \text { A pior dor possível }\end{array}$

Fonte: HARDIE, 2002.

A escala de contagem variável é o método que pode mesclar as variáveis fisiológicas e as alterações comportamentais. A somatória deste teste avalia a analgesia pelo menor valor (TEIXEIRA, 2005, apud ESPER, 2005).

\section{TRATAMENTO E CONTROLE DA DOR}

A dor apresenta aspectos negativos, tanto nos humanos quanto nos animais, podendo ser reconhecida pela ativação da resposta ao estresse. Geralmente a cura da lesão é prejudicada pela presença da dor, pois o paciente apresenta aumento do consumo de energia e diminuição da ingestão de alimento (energia), tornando-se mais propenso a um balanço energético negativo (HELLEBREKERS, $2002 \mathrm{~b}$ ).

Os processos dolorosos acarretam uma série de alterações fisiológicas que podem ser gravemente nocivas à saúde. A dor causa interferências nos eixos neuroendócrinos levando ao aumento nos níveis de aldesterona, cortisol e cotecolaminas. O aumento do cortisol induz à retenção de sódio e desbalanço hidroeletrolítico. Níveis elevados de cortisol geram hiperglicemia e o aumento das catecolaminas é responsável por alterações cardíacas como arritmias e aumento do consumo de oxigênio pelo miocárdio (FANTONI; MASTROCINQUE, 2002). 
BRAGA, N.S. e SILVA, A.R.C. Acupuntura como opção para analgesia em veterinária. PUBVET, Londrina, V. 6, N. 28, Ed. 215, Art. 1435, 2012.

As alterações da dor estão relacionadas principalmente com o estado emocional do animal, e também prejudicam os processos de cicatrização, liberam radicais livres, afetando assim a recuperação do paciente (ABIMUSSI et al., 2008).

Alguns autores, no entanto, afirmam que a presença da dor possui qualidades benéficas que podem ser perdidas após se estabelecer o controle da dor, pois quando o animal exibe sintoma de dor torna-se mais fácil reconhecer e diagnosticar certas afecções. Apesar disso deve-se ter em mente que muitos aspectos negativos podem ser atribuídos com a presença de dor prolongada, como já foi descrito anteriormente, pois a dor afeta o animal, tanto no sentido físico como no psicológico. Portanto é necessário que se obtenha o alivio da dor de forma que ela não seja totalmente eliminada, mas se torne muito mais suportável (FANTONI; MASTROCINQUE, 2002).

Fármacos, técnicas ou métodos usados para prevenir e controlar a dor devem ser adaptados individualmente para cada animal, baseando-se nos graus de trauma tecidual, características do comportamento individual, graus de dor e estado de saúde (AMERICAN VETERINARY MEDICAL ASSOCIATION, 2001 apud ANTUNES et al., 2008).

Alguns métodos podem ser utilizados para se obter analgesia. $\mathrm{Na}$ Medicina Veterinária, tem-se o emprego de drogas analgésicas que bloqueiam a transmissão nervosa (DRAEHMPAEHL; ZOHMANN, 1997). Porém, como já foi descrito anteriormente, esses fármacos podem apresentar efeitos indesejáveis e riscos aos animais (FANTONI; MASTROCINQUE, 2002).

As drogas analgésicas mais utilizadas em animais são os opióides, os anestésicos locais, os antiinflamatórios não-esteroidais a-2 agonistas, os agentes dissociativos, os agentes inalatórios e os benzodiazepínicos (LASCELLES, 2002). 
BRAGA, N.S. e SILVA, A.R.C. Acupuntura como opção para analgesia em veterinária. PUBVET, Londrina, V. 6, N. 28, Ed. 215, Art. 1435, 2012.

\section{ACUPUNTURA NA MEDICINA VETERINÁRIA}

A Acupuntura é uma forma terapêutica que pertence à Medicina Tradicional Chinesa (MTC) juntamente com técnicas de massagem (Tui-Na), exercícios respiratórios (Chi-Gung), orientações nutricionais (Shu-Shieh) e a farmacopéia chinesa (medicamentos de origem animal, vegetal e mineral) (XIE; PREAST 2007).

Os primeiros indícios de seu uso, tanto nos homens como nos animais, datam de cinco mil anos atrás. A primeira referência escrita de maior importância no ocidente, a respeito da acupuntura, data de 2700 aC.. No entanto, foi introduzida no Ocidente por jesuítas no século XVIII, porém apenas nas décadas de 1920 e 1930 foi divulgada pelo frânces Souliet de Morant e seus discípulos a partir da França (LUNA, 2002).

No Brasil, a acupuntura Veterinária, foi introduzida em 1950, pelo professor Frederico Spaeth, sendo fundada em 1958, a Associação Brasileira de Acupuntura. Em 1974, foi fundada a Sociedade Internacional de Acupuntura Veterinária e no Brasil a Acupuntura Veterinária difundiu-se principalmente a partir da década de 1980, tendo sido fundada a Associação Brasileira de Acupuntura Veterinária (ABRAVET), em 1999 (LUNA, 2002).

As bases filosóficas da Acupuntura estão contidas nas teorias gerais do Taoísmo sendo: Yin e Yange Cinco Movimentos ou Wu Xing (SCOGNAMILLOSZABÓ; BECHARA, 2010).

Segundo a MTC, a doença é resultado da interação entre o agente causal e o indivíduo, resultando em desequilíbrio nos componentes Yin e Yang do organismo. Essa desarmonia determina o curso da doença e está relacionada à oposição dos dois fatores: Energia Correta (ZhengQi) - fator intrínseco que traduz a resistência à doença - e Energia Perversa (XieQi) - o fator patogênico propriamente dito (SCOGNAMILLO-SZABÓ; BECHARA, 2010).

O termo Acupuntura se origina do latim (acus - agulha; pungare perfurar). Porém esta palavra não traduz a expressão chinesa "ShenShiu" (ZhenJiu) para esta terapia, que quer dizer espetar e queimar. A acupuntura 
BRAGA, N.S. e SILVA, A.R.C. Acupuntura como opção para analgesia em veterinária. PUBVET, Londrina, V. 6, N. 28, Ed. 215, Art. 1435, 2012.

reúne na verdade duas técnicas: a estimulação de áreas definidas na pele por agulhas e/ou por transferências de calor para fins terapêuticos. A natureza do tratamento pela acupuntura restabelece o equilíbrio entre estados de função contraditórios e com isso restaura a homeostase. Com esta terapia, há, portanto, a restituição da função normal do organismo quando em estados vegetativos alterados, como: tônus simpático aumentado (representado pelo Yang, por exemplo, na contratura muscular), ou um tônus parassimpático aumentado (representado por Yin, por exemplo diarréia) (DRAEHMPAEHL; ZOHMANN, 1997).

A acupuntura é utilizada, segundo a MTC para aumentar ou diminuir o fluxo de energia ( $Q i)$ de linhas ou canais, chamados de "meridianos". Embora não existam evidências da existência dos meridianos, a teoria da existência dos mesmos é amplamente enunciada como uma explicação para muitas das práticas da medicina tradicional chinesa (GAYNOR, 2000).

O efeito da acupuntura se dá por uma associação de mecanismos neurológicos e humorais. Os meridianos da acupuntura normalmente se localizam ao longo dos nervos periféricos e os acupontos estão proximamente relacionados às terminações nervosas. O efeito vai depender de receptores nervosos periféricos e de uma comunicação destes com o Sistema Nervoso Central (LUNA, 2002).

O acuponto é definido como um ponto da pele de sensibilidade espontânea ao estímulo e à resistência elétrica reduzida. Possui um diâmetro de $0,1 \mathrm{a} 5 \mathrm{~cm}$, entretanto é uma área de condutividade elétrica amplamente aumentada comparada às áreas da pele ao redor (SCHWARTZ, 2008 apud TAFFARES; FREITAS 2009). Estes estão localizados próximos a articulações, bainhas tendíneas, vasos, nervos, septos intramusculares, na ligação músculotendínea, nos locais de maior diâmetro do músculo e nas regiões de penetração dos feixes nervosos da pele (DRAEHMPAHEL; ZOHMANN, 1997).

Segundo LUNA (2002), quando se introduzem agulhas nesses pontos, ocorre uma liberação de bradicininas, histaminas, substancia P, leucotrienos, prostaglandinas e fator de ativação plaquetária, entre outras substâncias. 
BRAGA, N.S. e SILVA, A.R.C. Acupuntura como opção para analgesia em veterinária. PUBVET, Londrina, V. 6, N. 28, Ed. 215, Art. 1435, 2012.

Além da acupuntura tradicional corporal existem outros métodos que são: auriculoterapia, eletroacupuntura, hipoalgesia (analgesia por acupuntura), eletroacupuntura, terapia a laser, moxibustão, terapia neural, acuinjeção, escarificação da pele e o uso de ventosas com, ou sem a presença de agulhas. A hipoalgesia é o procedimento no qual se estimulam pontos de acupuntura constantemente por via manual ou elétrica (variação de voltagem) com o objetivo de se obter a analgesia, ou seja, hipoalgesia(DRAEHMPAEHL; ZOHMANN, 1997).

\section{MECANISMOS DE AÇÃO DA ACUPUNTURA NA DOR}

As estimulações, através da acupuntura, dos pontos proximais e distais ao local da dor restauram o equilíbrio e normalizam a causa da dor. Um dos princípios básicos e mais importantes envolve escolher pontos proximais e distais à área afetada (KLIDE; GAYNOR, 2006 apud TAFFARES; FREITAS, 2009).

A acupuntura atua sobre o controle da dor por ativação de vias opióides e não opióides. A estimulação promovida por essa técnica ativa o sistema modulador da dor por hiperestimulação das terminações nervosas de fibras mielínicas A-delta, responsáveis pela condução do estímulo aos centros medulares, encefálicos e eixo hipotálamo-hipofisário. Na medula espinhal, a modulação dos estímulos nociceptivos se dá por inibição pré-sináptica, devido à liberação de encefalinas e dinorfinas. No mesencéfalo, as encefalinas e a ativação do sistema central de modulação da dor resultam na liberação de serotonina e norepinefrina nos sistemas descendentes (Figura 4)(SANTOS; MARTELETE, 2004 apud TAFFARES; FREITAS, 2009). 


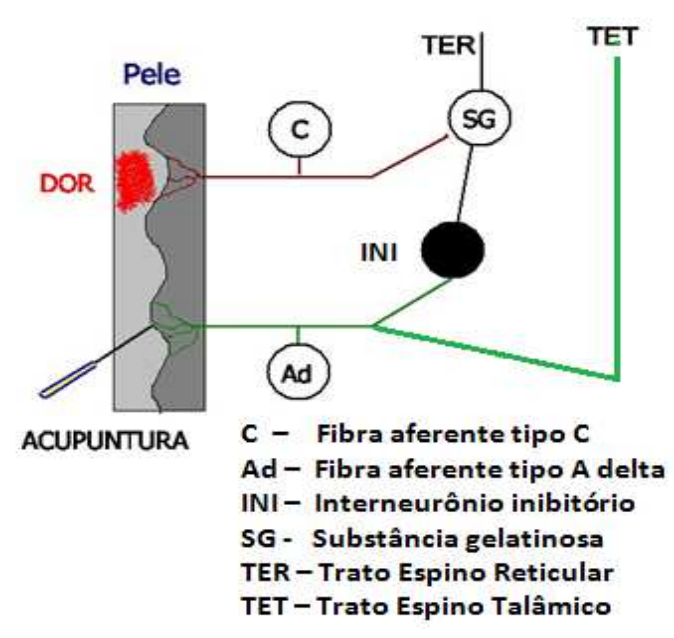

Figura 4.Acupuntura age como estímulo nociceptivo estimulando a fibra A delta, que ativa o interneurônio inibitório que libera encefalina. A encefalinabloqueia, na Substância Gelatinosa, a transmissão do sinal da dor conduzido pelas fibras tipo " $\mathrm{C}$ " para os tratos ascendentes da medula (TER).Por outra via ascendente, o trato espinotalâmico, o estímulo da fibra A delta é conduzido ao córtex cerebral, onde são interpretadas, ou "percebidas" as sensações de peso, distensão, calor ou parestesia que ocorrem durante o estímulo por acupuntura.

Fonte: Modificado de KONO, 2011.

KOO et al. (2008), ao estudarem os efeitos analgésicos da eletroacupuntura em ratos submetidos à torção do tarso, concluíram que a eletroacupuntura ativa neurônios bulboespinhais, o que resulta na liberação de noradrenalina e ativação de a2-adrenoreceptores do corno dorsal da medula, confirmando uma via não opióide de ação da eletroacupuntura.

A alta freqüência de estimulação elétrica (100-200 Hz) na eletroacupuntura induz a liberação de serotonina, adrenalina, noradrenalina, assim induzindo analgesia (GAYNOR, 2000). 
BRAGA, N.S. e SILVA, A.R.C. Acupuntura como opção para analgesia em veterinária. PUBVET, Londrina, V. 6, N. 28, Ed. 215, Art. 1435, 2012.

Em resumo, a acupuntura estimula a liberação de serotonina, substâncias opióides, e outros transmissores em vários níveis, reduzindo assim a transmissão e percepção da dor(GAYNOR, 2000).

\section{ACUPUNTURA COMO TRATAMENTO DA DOR}

A acupuntura para efeito analgésico é indicada em casos de dor visceral, dor cutânea, dor muscular traumática aguda, síndrome da dor miofascial, síndrome dolorosa na extremidade anterior, síndrome dolorosa na região da coluna vertebral, síndrome dolorosa na região de coxal e fêmur, terapia adjuvante para melhoria da analgesia, em caso de procedimentos cirúrgicos (pré, trans e pós-operatório), método complementar para analgesia em pacientes com câncer, entre outros (DRAEHMPAHEL; ZOHMANN, 1997).

Existem alguns métodos de estimulação dos pontos de acupuntura que são utilizados para obtenção da analgesia como técnica de agulhamento simples, eletroacupuntura, acuinjeção, moxibustão, laserpuntura, acupressão e implantes. (LUNA, 2002).

A acupuntura por agulhamento é uma técnica onde a agulha é inserida no acuponto desejado e atravessa a derme atingindo o tecido subcutâneo, podendo alcançar músculos ou ossos. Existe uma grande variedade no tamanho das agulhas, bem como no procedimento de inserção e de manipulação. O material mais utilizado é o aço inoxidável e agulhas intradérmicas também podem ser usadas, especialmente em acupontos no pavilhão auricular (auriculoterapia) (XIE; PREAST, 2007).

Os pontos da orelha também podem ser utilizados para efeito analgésico, já que refletem os órgãos do corpo. Após a colocação das agulhas na auriculoterapia, elas são fixadas com esparadrapo e retidas por um período que pode variar de um dia a uma semana. Essa técnica é pouco executada em animais (XIE; PREAST, 2007).

Na Medicina Veterinária, a eletroacupuntura (Figura5, figura 6) tem sido utilizada com fins de obtenção de hipoalgesia, devido à dificuldade de 
BRAGA, N.S. e SILVA, A.R.C. Acupuntura como opção para analgesia em veterinária. PUBVET, Londrina, V. 6, N. 28, Ed. 215, Art. 1435, 2012.

estimulação manual da agulha nos pacientes. A intensidade da estimulação é regulada de acordo com o limite individual da dor do paciente, manifestado por estremecimentos rítmicos da pele e dos músculos adjacentes. A frequência e a tensão elétrica devem ser variadas, pois muitas vezes o paciente acostuma-se a essa estimulação. Além disso, o alcance da hipoalgesia é influenciado pela escolha dos acupontos e da forma de estimulação (LUNA, 2002).

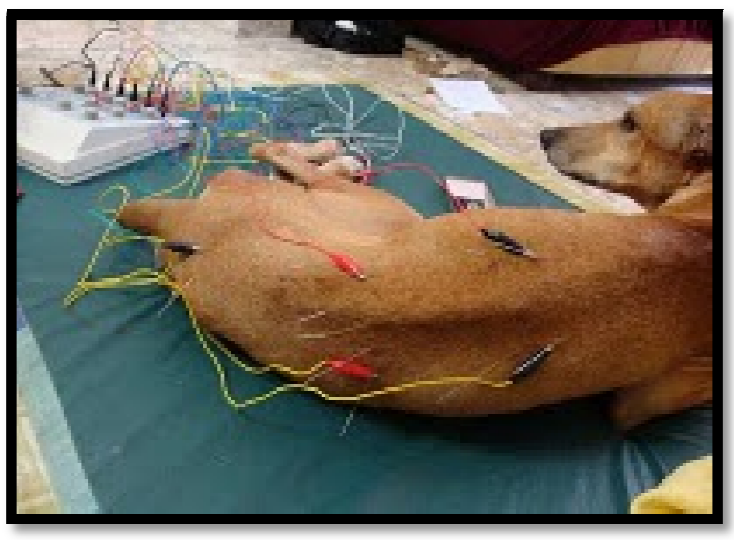

Figura 5. Uso de aparelho elétrico que, conectado às agulhas, transmite estímulos aos pontos de acupuntura, potencializando seu efeito e/ou produzindo analgesia Fonte: ACUPETS, 2011.

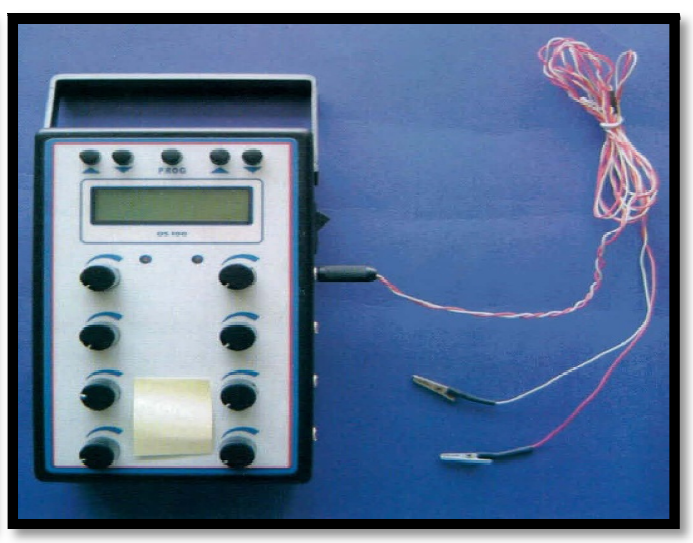

Figura 6. Aparelho de eletroestimulação utilizado para a eletroacupuntura percutânea. Fonte: HAYASHI; MATERA, 2005

Assim, a escolha dos pontos deve ocorrer de acordo com o percurso do meridiano e a distribuição dos nervos que atravessam a área de acontecimento da dor. Utiliza-se eletroacupuntura de alta frequência para analgesia de pontos do tronco e de baixa frequência para pontos dos membros (DRAEHMPAHEL; ZOHMANN, 1997).

A eletroacupuntura também vem sendo usada para produzir hipoalgesia para realização de vários processos cirúrgicos nos animais domésticos (LUNA, 2002).

Para a utilização da eletroacupuntura, primeiro é feita a introdução da agulha no animal e nas agulhas adaptam-se os grampos do aparelho 
BRAGA, N.S. e SILVA, A.R.C. Acupuntura como opção para analgesia em veterinária. PUBVET, Londrina, V. 6, N. 28, Ed. 215, Art. 1435, 2012.

desligado, iniciando-se com voltagem zero e aumentando-se lentamente. A intensidade da corrente deve ser aumentada lentamente até que o animal apresente desconforto, quando então é reduzida. O período de latência é de em torno de 20 a 30 minutos (LUNA, 2002).

A acuinjeção nada mais é do que a aplicação de fármacos nos pontos de acupuntura, podendo ser também utilizados produtos homeopáticos, produtos fitoterápicos e soluções fisiológicas.A cuinjeção é especialmente indicada nos pontos auriculares. Também pode ser empregada para tratar lombalgias, melhorando a excreção de toxinas metabólicas e substâncias álgicas pela vias linfáticas (DRAEHMPAHEL; ZOHMANN, 1997). A vitamina B12 também pode ser utilizada na acuinjeção (HALTRECHT, 1999).

Moxabustão também é um método da MTC onde a moxa (Figura7) (Artemisia vulgares) ou outras ervas (canela, gengibre seco, cravo, mirra, e incenso). Esse material libera calor de maneira uniforme. Existem dois métodos gerais de moxabustão: osdiretos e os indiretos. No método direto os cones de moxa são colocados diretamente sobre o ponto de acupuntura e posteriormente acendidos, porém esse método não é muito utilizado em animais visto que eles não permanecem quietos durante o procedimento, além de que esse método pode provocar queimaduras. Já o método indireto pode ser realizado através de bastões de moxa inteiros que são mantidos sobre o ponto de acupuntura com uma distância de 0,5 a $1 \mathrm{~cm}$ da pele, ou que são colocados sobre agulhas de acupuntura inseridas no animal. A aplicação deste método tem duração de cinco a dez minutos (XIE; PREAST 2007). 


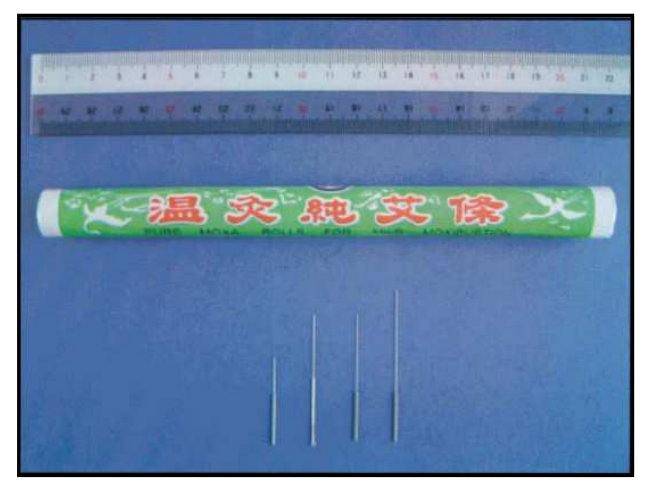

Figura 7. Bastão da erva Artemísia vulgarise agulhas de acupuntura de diversos tamanhos.

Fonte: HAYASHI; MATERA, 2005

A laserpuntura utiliza irradiação de softlaser nos pontos de tratamento usual de acupuntura no corpo e na orelha. É um método não invasivo e o tempo de estimulação varia de 30 segundos a umminuto. O laser age aumentando a síntese de adenosina trifosfato(ATP) na célula, levando a uma hiperpolarização e bloqueio de estímulos menores, assim a transmissão de estímulos da dor é restringida levando uma redução da mesma (DRAEHMPAHEL; ZOHMANN, 1997).

A acupressão é um método que consiste na aplicação de pressão sobre a superfície do corpo de uma forma geral (massagem) ou em pontos específicos. Normalmente não é utilizada por médicos veterinários, mas os proprietários podem ser treinados para utilizar essa técnica como forma de complementar à terapia com agulhas (ALTMAN, 2006 apud TAFFAREL; FREITAS, 2009).

Vários materiais podem ser implantados no tecido próximo ao ponto de acupuntura para obter um estímulo mais prolongado. Catgut ou outros materiais de sutura podem ser implantados nos pontos de acupuntura com este propósito. No entanto, o material mais utilizado é ouro em forma de contas esterilizadas. A técnica pode ser utilizada para tratamento de osteoartrite (especialmente displasia coxo-femural), epilepsia e doença do disco intervertebral (HALTRECHT, 1999). 
BRAGA, N.S. e SILVA, A.R.C. Acupuntura como opção para analgesia em veterinária. PUBVET, Londrina, V. 6, N. 28, Ed. 215, Art. 1435, 2012.

Independentemente dos métodos escolhidos, a acupuntura no tratamento da dor consta do estímulo de pontos anatômicos específicos, como por exemplo, os apresentados nas figuras 8 e 9,produzindo efeitos terapêuticos e analgésicos. Ela não elimina completamente a dor, mas produz hipoalgesia. A experiência clínica mostra que a acupuntura pode ser utilizada como única ferramenta no controle da dor em alterações musculoesqueléticas, mas não no pós-operatório (LUNA, 2002).

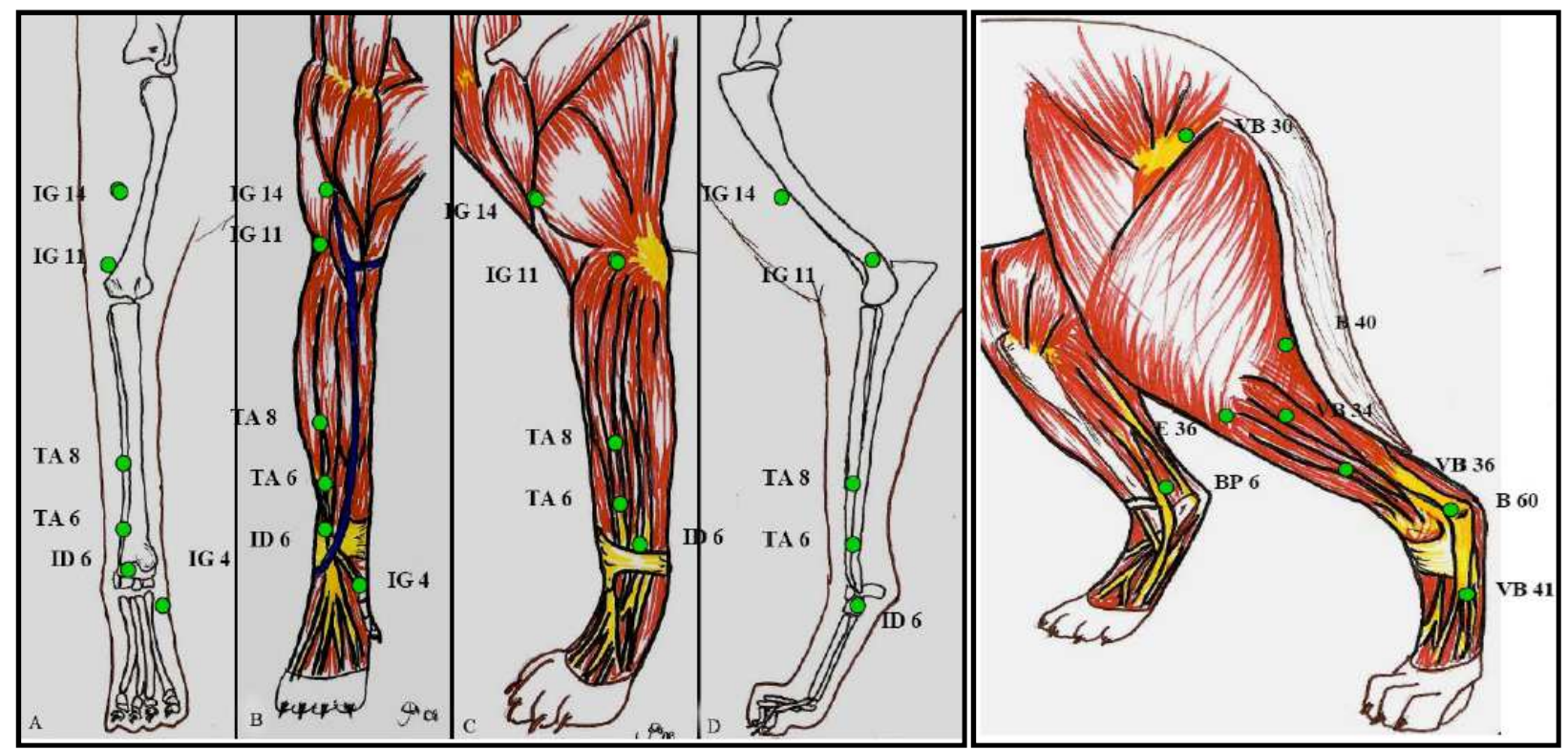

Figura 8.Desenho esquemático de membro Figura 9. Desenho esquemático torácico direito de cão em vista cranial (A e B) e lateral ( $C$ e D), evidenciando a localização dos acupontos IG 14, IG 1, IG 4, TA 6, TA 8 e ID 6. Fonte: TAFFAREL; FREITAS, 2009. de membro pélvico de cão em vista lateral esquerda, evidenciando a localização anatômica dos acupontos BP 6, E 36, VB 30, VB 34, VB 36, VB 41 , B 40 e B 60 .

Fonte: TAFFAREL; FREITAS, 2009. 
BRAGA, N.S. e SILVA, A.R.C. Acupuntura como opção para analgesia em veterinária. PUBVET, Londrina, V. 6, N. 28, Ed. 215, Art. 1435, 2012.

A localização dos pontos pode ser feita de acordo com a descrição anatômica e topográfica dos mesmos, através de palpação (pontos dolorosos, edemaciados ou com elevações), ou através de um aparelho de localização dos pontos que detecta os acupontos no animal (DRAEHMPAHEL; ZOHMANN, 1997).

Os animais devem ser posicionados adequadamente, em decúbito lateral ou em estação, e contidos com ajuda do proprietário, pois raramente é usada a contenção química. Procede-se à inserção das agulhas, ou a escolha de outro método de estímulo, sendo a duração do evento entre 20 e 30 minutos (LOONEY, 2000 apud HAYASHI; MATERA, 2005).

Acupuntura pode ser aplicada a cada dois a três dias em casos agudos e, em casos crônicos, uma vez por semana durante quatro a oito semanas. Quando o quadro se estabiliza, pode-se diminuir a frequência a intervalos quinzenais. Posteriormente, avalia-se o paciente, a cada três a seis meses, sendo aconselhada essa avaliação em período de estação mais quente ou fria do ano, dependendo do problema do animal (HALTRECHT, 1999).

Gakiya (2009) realizou um estudo na Universidade do Oeste Paulista, onde o objetivo era avaliar os efeitos cardirespiratório, sedativo e analgésico pós-operatório, mediados pela aplicação de morfina, eletroacupuntura ou pontos falsos de acupuntura em cadelas submetidas à mastectomia parcial ou total. Foram avaliadas 30 cadelas sem raça definida, adultas que foram divididas em três grupos. O grupo Gmorf $(n=10)$ foi administrado morfina no momento da medicação pré-anestésica (MPA), no grupo GEA $(n=10)$ foi realizada aeletroacupuntura30 minutos após MPA. No grupo Gsham $(n=10)$ foi aplicada eletroacupuntura30 minutos após MPA, só que as agulhas foram colocadas em pontos falsos (regiões próximas dos pontos de acupuntura não percorridas pelos meridianos). Através desse trabalho foi possível observar que a frequência cardíaca em todos os grupos, durante o procedimento cirúrgico, se manteve estável; a administração preventiva de morfina induziu efeito sedativo mais evidente na primeira hora de avaliação pós-operatória, em relação aos animais tratados com eletroacupuntura; a estimulação elétrica de 
BRAGA, N.S. e SILVA, A.R.C. Acupuntura como opção para analgesia em veterinária. PUBVET, Londrina, V. 6, N. 28, Ed. 215, Art. 1435, 2012.

alguns acupontos conferiu analgesia superior, permitindo a redução do requerimento analgésico no período pós-operatório, quando comparado à administração preventiva de morfina e agulhamento de pontos falsos de acupuntura, de acordo com os pesquisadores que conduziram este trabalho.

Em outro estudo, realizado por Hayashi (2006), na Faculdade de Medicina Veterinária e Zootecnia da Universidade de São Paulo, o objetivo era avaliar a eficácia da acupuntura no tratamento de discopatia intervertebral toraco-lombar em cães. Nesse estudo foram avaliados 50 cães de diversas raças (Dachshund, Poodle, Cocker Spaniel, Pequinês, Springer Spaniel, Lhasa Apso) e sem raça definida, sendo divididos em dois grupos. O grupo I constava de 26 cães que foram tratados com acupuntura (agulhamento e eletroacupuntura) com associação de tratamento médico, e o grupo II, com 24 animais que só receberam tratamento médico. A discopatia toracolombar promove estados dolorosos muitas vezes debilitantes, caracterizando sinais neurológicos variáveis (leve hiperestesia da região, paraparesia de graus variados, paraplegia, alteração da miç̧ão, perda da locomoção e perda da percepção de dor profunda).De acordo com autor, foi possível concluir que a acupuntura pode ser aplicada associada a tratamento médico em cães com discopatia toraco-lombar, antecipando a locomoção e melhora na evolução neurológica em cães que ainda apresentavam percepção a dor profunda, mas tinham déficit locomotor em membros pélvicos.

Saletu et al. (1975) confirmaram que a eletroacupuntura é mais efetiva para o tratamento de dor crônica, quando comparada com a acupuntura tradicional (por agulhamento). E pesquisas recentes, como as apresentadas na revisão bibliográfica publicada por Cassu; Luna (2004) citada por Cumán (2009),demonstraram uma taxa de sucesso superior a $70 \%$ para o tratamento de dor crônica em pacientes com osteoartrites, desconforto miofacial, dores na coluna e dores de cabeça.

Quando a acupuntura é usada por um profissional treinado, é improvável que cause efeitos negativos. A decisão em se utilizar essa técnica geralmente é baseada na questão de seu benefício ou não para o animal. Em algumas 
BRAGA, N.S. e SILVA, A.R.C. Acupuntura como opção para analgesia em veterinária. PUBVET, Londrina, V. 6, N. 28, Ed. 215, Art. 1435, 2012.

situações pode haver contraindicações, como em fêmeas gestantes e a eletroacupuntura não deve ser utilizada em animais com doenças cardíacas, nem em estado de extrema fraqueza ou fadiga. Porém, a acupuntura ainda pode ser aplicada nestes pacientes com a utilização de técnicas adequadas nesses casos (HALTRECHT, 1999).

\section{CONSIDERAÇÕES FINAIS}

O conhecimento da fisiopatologia da dor é fundamental para o entendimento dos mecanismos desencadeantes dos processos dolorosos, sejam fisiológicos ou patológicos.

O reconhecimento da dor é essencial para a instituição de uma terapia analgésica eficiente já que a dor, assim como foi apresentado por Hellebrekers (2002a), é uma forma de prejudicar o bem-estar, pois a síndrome dolorosa pode ser considerada uma doença, gerando alterações na homeostasia orgânica que implicam em perda em qualidade de vida para o paciente. Talvez seja interessante envolver o proprietário e os veterinários na detecção e se possível, quantificação da dor, a fim de que se criem mecanismos mais efetivos para tratá-la e avaliar o efeito do tratamento, pois conforme Ford, Mazzaferro, (2007), a caracterização e o reconhecimento da dor em animais são um desafio, não existindo também formas para tratá-la com especificidade.

A acupuntura mostra-se como uma alternativa de terapia adjuvante no controle da dor, já que tem poucas contraindicações. A hipoalgesia através da acupuntura prova ser efetiva no tratamento de dores agudas e crônicas. Pelo exposto neste trabalho e pelo pequeno número de pesquisas com este enfoque, seria interessante que mais estudos específicos fossem desenvolvidos nesta área, visando o aumento de alternativas balizadas para o controle da dor em animais e a melhora nas suas condições de bem-estar. 


\section{REFERÊNCIAS}

ABIMUSSI et al. Analgesia preventiva no tratamento da dor e na recuperação pós-operatória de amputação alta de membro torácico. Veterinária e Zootecnia. v.15, n.2, p. 29 ago. 2008.

ACUPETS. Acupuntura Veterinária, 2011.Disponível em: http://acupets.blogspot.com/p/algunscasos.html. Acessado em: 04/10/2011.

ALMEIDA T. P.et al.Classificação dos processos dolorosos em medicina veterinária. Veterinária em Foco. Canoas - RS. v.3, n.2, p. 107 - 116, jan./jun. 2006.

BROOM, D.M. Indicators of poor welfare.British Veterinary Journal.London, v.142, p.524$526,1986$.

CAPONERO, R.; VIEIRA, D. E.; TEIXEIRA, M. J. Dor no Doente com Câncer. Prática Hospitalar, $2001 . \quad$ Disponível em: http://www.praticahospitalar.com.br/pratica\%2035/paginas/materia\%2001-35.html. Acessado em: 03/05/2011.

COGGESHALL, R. E. et al. Discharge characteristics of fine medial articular afferents at rest and during passive movements of inflamed knee joints. Brain Research. v. 272, p. 185-188, 1983.

CUMÁN, B. D. N. Eletroacupuntura no controle da dor. Porto Alegre, Universidade federal do Rio Grande do Sul, 2009. 44 f. Monografia de conclusão de curso Medicina Veterinária, Universidade Federal do Rio Grande do Sul, Porto Alegre, 2009.

DRAEHMPAEHL D.; ZOHMANN A. Acupuntura no Cão e no Gato: Princípios Básicos e Prática Científica. $1^{\text {a }}$ ed. São Paulo-SP: ROCA, 1997 p. 34-37.

ESPER G. V. Z. Estudo comparativo do emprego da eletroacupuntura e cloridrato de meperidina na analgesia de cadelas submetidas à castração cirúrgica. Curitiba, Universidade Federal do Paraná, 2005. 95 f. Dissertação (Pós-Graduação em Medicina Veterinária para obtenção de titulo de Mestre) Programa de Pós-Graduação em Ciências Veterinárias, Universidade Federal de Curitiba, Curitiba, Paraná, 2005.

FANTONI, D. T.; MASTROCINQUE, S. Fisiopatologia e controle da dor. In: FANTONI, D. T.; CORTOPASSI, S. R. G. Anestesia em cães e gatos. São Paulo: Roca, p. 323-336, 2002

FIVE FREEDOMS.Farm Animal Welfare Council, 2009.Disponível em: <http://www.fawc.org.uk/freedoms.htm>. Acessado em 20/05/2011

FORD, R. B.; MAZZAFERRO, E. M. Segundo Kirk e Bister - Manual de Procedimentos Veterinários e Tratamento Emergencial. ROCCA, Rio de Janeiro - RJ, 2007. 747p

GAKIYA, H. H. Estudo comparativo entre o uso da eletroacupuntura e morfina para o controle da dor pós-operatória em cadelas submetidas à mastectomia. Presidente Prudente, Universidade do Oeste Paulista, 2009. 62 f. Dissertação para obtenção do titulo de Mestrado em Ciência Animal, Universidade do oeste Paulista, São Paulo, 2009.

GAYNOR, J. S. Acupuncture for management of pain. Veterinary Clinics of North America: Small Animal Practice.v. 30, n. 04, p. 875-884, july. 2000. 
HALTRECHT, H. Veterinary acupuncture. Canadian Veterinary Journal, v. 40, p. 401-403, 1999.

HAYASHI, A. M.; MATERA, J. M. Princípios gerais e aplicações da acupuntura empequenos animais: revisão de literatura. Revista de Educação Continuada - CRMV.SP, São Paulo, v. 8, n. 2, p. 109-122,2005.

HAYASHI, A. M. Estudoclinico da eficácia da acupuntura no tratamento da discopatia intervertebral traço-lombar em cães. São Paulo, Faculdade de Medicina Veterinária e Zootecnia da Universidade de São Paulo, 2006. 105 f. Dissertação para obtenção do titulo de Mestrado em Medicina Veterinária, Faculdade de Medicina Veterinária e Zootecnia da Universidade de São Paulo, 2006.

HARDIE, E. M. Reconhecimento do comportamento doloroso em animais. In: HELLEBREKERS L. J. Dor em animais. Barueri: Manole, 2002. p.49-68.

HELLEBREKERS, L. J. A Dor em animais In:

Dor em animais. Barueri: Manole, 2002a.p. 11-15.

HELLEBREKERS, L. J. Fisiopatologia da dor em animais e sua consequência para terapia analgésica In: Dor em animais. Barueri: Manole, 2002b. p. 69-78.

KLAUMANN, P. R.; et al. Patofisiologia da dor. Archives of Veterinary Science. v. 13, n.1, p.1-12, 2008.

KONO, A. Kono Acupuntura: Mecanismo de Ação, 2011. Disponivel em: http://pontoacupuntura.blogspot.com/2011_04_01_archive.html. Acessado em 12/10/2011.

KOO, S. T.; Electroacupuncture-induced analgesia in a rat model of ankle sprain pain is mediated by spinal alpha-adrenoceptors. Pain, v.135, n.1-2, p.11-19, 2008.

LAMONT A. L.; TRANQUILI W. J.; GRIMM K. A. Physiology of pain. Veterinary Clinics of North America: Small Animal Practice. v. 30, n. 04, p. 703-728, july 2000.

LASCELLES, B. D. X. Farmacologia clínica de agentes analgésicos. In: HELLEBREKERS L. J. Dor em animais. Barueri: Manole, 2002. p.81-108.

LUNA, S. P. L. Emprego da Acupuntura em anestesia In: FANTONI, D. T.; CORTOPASSI, S. R. G. Anestesia em cães e gatos. São Paulo: Roca, p. 323-336, 2002

RAMOS, R. J. Bem-estar animal: A ciência de respeito aos animais. Informativo do Instituto Ecológico Aqualung. n. 68, ano XII, jul-ago 2006.

SCOGNAMILLO-SZABÓ, M. V. R.; BECHARA, G. H. Acupuntura: histórico, bases teóricas e sua aplicação em Medicina Veterinária. Ciência Rural. v.40, n.2, p.491-500, fev, 2010

TAFFAREL, M. O.; FREITAS, P. M. C.Acupuntura e analgesia: aplicações clínicas e principais acupontos. Ciência Rural.v. 39, n.9, jun, 2009.

XIE, H.; PREAST, V. Xie's veterinary acupuncture.Oxford: Blackwell, 2007. 376p.

WSPA. Victoria Braithwaite fala sobre senciência animal, Jun 17, 2010. Disponível em:<http://www.wspabrasil.org/latestnews/2010/Victoria-Braithwaite-fala-sobre-sencienciaanimal.aspx>. Acessado em 20/09/2011 